Journal für Mobilität und Verkehr

ISSN 2628-4154

www.dvwg.de

\title{
Was hindert Länder im südlichen Afrika an einer weiteren Verbesserung der Supply Chain- Leistung?
}

\author{
Stephan Hofmann* \\ HPC Hamburg Port Consulting GmbH, Ballinkai 1, 21129 Hamburg, Deutschland
}

\section{Abstract}

Die Mehrheit der Länder im südlichen Afrika liegt weit unter ihrem Supply Chain-Potenzial. Zugleich ist die ökonomische Entwicklung in vielen Ländern in der breiten Bevölkerung gering. Ziel dieses Projektes war es, Engpässe von Supply Chain-Leistung zu identifizieren und Maßnahmen zu bewerten, um Supply Chain-Leistung und ökonomische Entwicklung in ausgewählten Ländern im südlichen Afrika steigern zu können. Dabei wurden ökonomische, politische, kulturelle, technische und organisatorische Faktoren berücksichtigt.

Schlagwörter/Keywords:

Engpässe, Logistik-Leistung, Supply Chain-Leistung, Südliches Afrika

\section{Engpässe von Supply Chain-Leistung im südlichen Afrika}

Seehäfen, Straßentransport, Schienentransport und Produktionsstandorte sowie ihre Koordinationsfunktionen (hier zusammengefasst als Supply Chains) im südlichen Afrika sind durch sehr unterschiedliche Leistungsgrade charakterisiert. Vergleiche der Supply Chain-Leistung von Ländern im südlichen Afrika zeigen, dass nur sehr wenige Länder als sogenannte "Supply Chain Performer" beschrieben werden können. Einige Länder können als "Upper" oder "Lower Partial Supply Chain Performer" beschrieben werden. Dann gibt es Länder, die als „Supply Chain Underperformer" beschrieben werden können (Arvis et al. 2016: 9-10, 38-41; UNIDO \& UNCTAD 2011: 43-51; WEF 2017: 326-332). Anders ausgedrückt, die Mehrheit der Länder liegt weit unter ihrem Supply Chain-Potenzial. Dies ist so trotz der Tatsache, dass Supply Chain-Leistung über die gelingende Einbindung einer Volkswirtschaft in internationale Wertschöpfungsnetzwerke entscheidet (Saslavsky \& Shepherd 2012). Die Einbindung in internationale Wertschöpfungsnetzwerke hat einen Einfluss auf die Gestaltung von lokalen Wertschöpfungsnetzwerken einer Volkswirtschaft. So kann Supply Chain-Leistung einen wesentlichen Beitrag zu ökonomischem Wachstum und ökonomischer Entwicklung eines Landes leisten. Gleichzeitig ist die ökonomische Entwicklung in vielen Ländern des südlichen Afrikas in der breiten Bevölkerung verhältnismäßig gering (UNDP 2016: 198-201).

\section{Supply Chain-Leistung und ökonomische Entwicklung}

Viele Berichte verdeutlichen, dass Logistik und Supply Chains einen positiven Beitrag zu ökonomischem Wachstum und zu ökonomischer Entwicklung eines Landes leisten können. So beschreiben die Autoren des Connecting to Compete Berichtes, dass Logistik eine der wesentlichen Beitragenden zur ökonomischen Entwicklung ist (Arvis et al. 2016: 1). Die Autoren des Global Competitiveness Report geben an, dass das Niveau der Produktivität, in anderen Worten die Wettbewerbsfähigkeit, als eine der Haupt-Determinanten von ökonomischer Entwicklung angesehen wird (WEF 2017: 11). Die Autoren des Atlas of Economic Complexity schreiben, dass eine Verbesserung der Produktionskomplexität ein verlässlicher Ansatz zur Erreichung von ökonomischer Entwicklung ist (Hausmann et al. 2013: 66). Das Verhältnis von Supply Chains auf der einen Seite und ökonomischer Entwicklung auf der anderen Seite ist jedoch deutlich komplexer als diese kurzen Aussagen suggerieren. Basierend auf bestehender Literatur hat diese Arbeit vier wesentliche Mechanismen identifiziert, über die Supply Chain-Leistung zu ökonomischer Entwicklung eines Landes beitragen kann:

\footnotetext{
* Korrespondierender Autor.

E-Mail: s.hofmann@hpc-hamburg.de (S. Hofmann)
} 
Dies sind (1) der Austausch von Gütern, (2) der Austausch von Wissen, (3) lokale Wertschöpfung und Integration in Wertschöpfungsnetzwerke sowie (4) externer Zusatznutzen und ökonomische Agglomeration. Aus einer nationalen Entwicklungsperspektive kann grundsätzlich gesagt werden, dass es wert ist, Supply Chain-Leistung einer Region oder eines Landes zu fördern. Jedoch müssen folgende Aspekte berücksichtigt werden. Erstens, wie im Fall von Handel, in Abhängigkeit von der konkreten Gestaltung von Supply Chains, kann Supply Chain-Leistung sowohl positive als auch negative Effekte auf ökonomisches Wachstum und ökonomische Entwicklung eines Landes haben. Zweitens, ob ökonomisches Wachstum angemessen in ökonomische Entwicklung übersetzt wird, hängt wesentlich auch von der Gestaltung von Institutionen, im Sinne von Regeln, eines Landes ab (cf. WEF 2017: 7).

\section{Ziel des Projektes}

Die Logistik- und Produktionsleistung von Ländern wird durch eine Vielzahl von Faktoren bestimmt. Während einige Faktoren eine Ausprägung haben können, die förderlich für Supply Chain-Leistung ist, können andere Faktoren eine Ausprägung haben, die hemmend auf Supply Chain-Leistung wirkt. Trotz positiver Beiträge von förderlichen Faktorausprägungen, können negative Beiträge die Supply Chain-Leistung begrenzen. Es könnte angenommen werden, dass in einem Land mit geringer Supply Chain-Leistung alle diese Faktoren eine geringe Leistung aufweisen und damit alle verbessert werden müssten, um die Supply Chain-Leistung zu steigern. Jedoch leisten verschiedene Faktoren einen unterschiedlichen Beitrag zur Supply Chain-Leistung (cf. Hausmann \& Klinger \& Wagner 2008: 17). Zudem mag es verlockend sein, alle Faktoren verbessern zu wollen, die die Supply Chain-Leistung bestimmen. Aufgrund der Vielzahl von Faktoren und begrenzten Ressourcen ist es jedoch unwahrscheinlich, dass dieser Ansatz zum Erfolg führt (cf. Hausmann \& Rodrik \& Velasco 2008: 329; UNIDO \& UNCTAD 2011: 34). Vielmehr sollten Maßnahmen der Verbesserung auf Faktoren gerichtet werden, die den effektivsten und effizientesten Einsatz von Ressourcen versprechen (Hall \& McCalla \& Comtois 2011: 1; Feige 2007: 25).

Ziel dieses Projektes war es, ausschlaggebende Engpässe von Supply Chain-Leistung zu identifizieren und Verbesserungsmaßnahmen zu bewerten, um schließlich Supply Chain-Leistung in ausgewählten Ländern im südlichen Afrika zu steigern. Die zentrale Frage dieser Arbeit war, was hindert Länder im südlichen Afrika an einer weiteren Verbesserung der Supply Chain-Leistung.

Dabei wurden sowohl ökonomische, politische, kulturelle, technische als auch organisatorische Faktoren als mögliche Engpassfaktoren in Betracht gezogen. Es wurden einerseits Maßnahmen von Supply Chain-Akteuren berücksichtigt, die einen unmittelbaren Einfluss auf die Supply Chain-Leis- tung haben; andererseits wurden Maßnahmen von Supply Chain-Akteuren berücksichtigt, die Maßnahmen von anderen Akteuren hervorrufen könnten. Zu den letzteren zählen insbesondere auch Maßnahmen des öffentlichen Sektors.

Der Umfang des Projekts wurde in zwei Dimensionen begrenzt; zum einen im geographischen und zum anderen im funktionalen Umfang. Der geographische Umfang wurde zunächst auf Länder auf dem Festland der Southern African Development Community (SADC) begrenzt. In einem Fallauswahlprozess wurden drei Länder als zentrale Fälle ausgewählt. Diese Länder weisen eine Reihe von gemeinsamen Ausprägungen in Faktoren auf, die Supply Chain-Leistung bestimmen; zugleich haben diese Länder große Unterschiede in der Supply Chain-Leistung. Diese Länder umfassen, als "Supply Chain Underperformer" Angola, als „Partial Supply Chain Performer" Namibia und als "Supply Chain Performer" Südafrika. Da die Supply Chain-Leistung von Nachbarländern die Leistung in diesen drei Küstenländern mitbestimmt und teilweise auch rechtfertigt, wurden Nachbarländer als periphere Fälle ergänzend in dieser Arbeit mitberücksichtigt.

Der funktionale Umfang wurde auf Seehäfen, Straßentransport, Schienentransport und Produktionsstandorte beschränkt. Lufttransport spielt zwar eine wichtige Rolle in Supply Chains im südlichen Afrika, blieb jedoch weitgehend unberücksichtigt, um sicherzustellen, dass der Fokus dieser Arbeit nicht beeinträchtigt wird. Gleiches gilt für Flüssigmassengüter im Pipelinetransport. Binnenwasserstraßen stellen zwar einen kosten-effizienten Verkehrsträger dar, sind aber selten im südlichen Afrika und damit von geringer Wichtigkeit hier.

\section{Vorgehen zur Identifizierung von Engpässen}

Es besteht bereits eine Vielfalt von Ansätzen und Berichten zum Vergleich von einzelnen Komponenten von Supply Chain-Leistung. Während sich einige Ansätze ausschließlich auf die Effizienz-Komponente von Leistung beschränken, berücksichtigen andere Ansätze - wenngleich in unterschiedlichem Ausmaß - sowohl die Effizienz als auch die Effektivitäts-Komponente von Supply Chain-Leistung. Ansätze, die beide Komponenten berücksichtigen, umfassen unter anderem den World Bank "Connecting to Compete"- und den „Doing Business"-Bericht, den Center for International Development der Harvard University „Atlas of Economic Complexity", den World Economic Forum „Global Competitiveness Report" und die World Bank "Worldwide Governance Indicators". Wenngleich diese fünf Berichte wichtige Start- und Referenzpunkte lieferten, waren sie jedoch nicht in der Lage, die zentrale Frage dieser Arbeit zu beantworten. Daher war es notwendig, ein methodisches Vorgehen zu gestalten, das es ermöglichte, die Engpässe zu identifizieren.

Ein Fallstudien-Forschungsdesign stellte die Grundlage dar. Die Annahme war, dass die ausgewählten Länder eine Reihe von gemeinsamen Ausprägungen in den Faktoren 
Abbildung 1: Vorgehen und Methoden

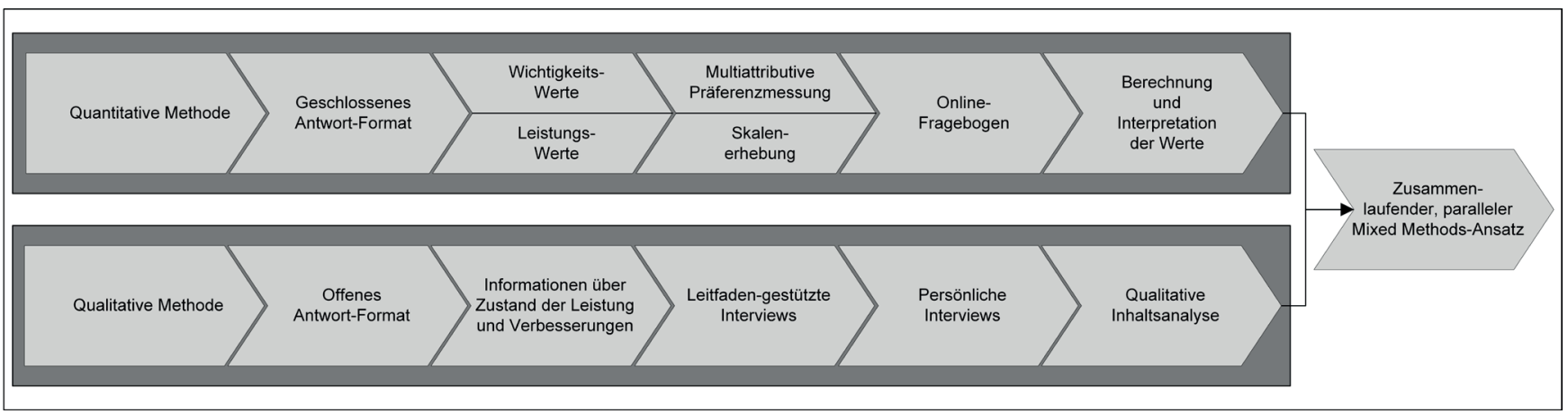

Quelle: Eigene Darstellung.

haben, die Supply Chain-Leistung bestimmen, zugleich jedoch starke Unterschiede in der Supply Chain-Leistung aufweisen. Eine quantitative Methode, das heißt ein Online-Fragebogen, und eine qualitative Methode, qualitative Interviews, wurden ausgewählt, um die notwendigen Informationen zu generieren. Mit Hilfe einer Conjoint-Analyse und Likert-Type-Skala ermittelte der Online-Fragebogen Wichtigkeits- und Leistungswerte von Bestandteilen von Supply Chain-Leistung. Basierend auf Leitfaden-gestützten Interviews erfragten die qualitativen Interviews Informationen hinsichtlich des Zustandes der Supply Chain-Leistung (u. a. Stärken, Schwächen, Ursachen) und möglichen Verbesserungen (u. a. Bereiche und Maßnahmen der Verbesserung, erwartete Verantwortlichkeiten, mögliche Hindernisse und Best-Practices). Dies erfolgte jeweils für die Bereiche Seehäfen, Straßentransporte, Schienentransporte und Produktionsstandorte. Beide Informationsstränge wurden schließlich in einer gemeinsamen Analyse zusammengeführt, wie in Abbildung 1 dargestellt.

Im Zeitraum von Mai 2016 bis Dezember 2016 wurden insgesamt 45 Fragebögen ausgefüllt und es wurden - vor allem persönlich vor Ort, aber auch telefonisch - 42 Interviews geführt. Die Daten und Informationen wurden mithilfe von statistischen Analyseanwendungen und der qualitativen Inhaltsanalyse ausgewertet.

\section{Engpässe von Supply Chain-Leistung in ausgewählten Ländern}

Die Ergebnisse zeigen die folgende Situation für die Länder Angola, Namibia und Südafrika:

\section{Angola}

Ein (1) Mangel an öffentlichen und privaten Devisen, (2) Defizite in dem Import-orientierten Transportsystem, (3) Defizite im inländischen Transportsystem, (4) Defizite in der Elektrizitätsversorgung sowie (5) Defizite im Bildungswesen und den Arbeitskräften hemmen die Supply Chain-Leistung in Angola. Das Land muss zunächst ein angemessenes Im- port-orientiertes und inländisches Transportsystem sowie eine angemessene allgemeine wirtschaftliche Infrastruktur erreichen, bevor es eine Markt-orientierte landwirtschaftliche und industrielle Produktionsindustrie aufbauen kann und damit die Wirtschaft diversifizieren kann.

\section{Namibia}

Die (1) geringe inländische Marktgröße, (2) Defizite im Schienentransportsystem, (3) Defizite im Bildungswesen und den Arbeitskräften, (4) Defizite in der Wasserversorgung, (5) Defizite in der Elektrizitätsversorgung sowie (6) Defizite in der Investitionspolitik hemmen die Supply Chain-Leistung in Namibia. Das Land muss sein inländisches und grenzüberschreitendes Transit-Transportsystem erhalten und stärken sowie produzierende Unternehmen anziehen, um wiederum höhere Transportvolumina zu erreichen.

\section{Südafrika}

Die (1) unzureichende Schienentransportleistung, (2) Defizite bei Grenzübergangsprozessen, (3) Defizite im Bildungswesen und bei den Arbeitskräften, (4) Defizite in der öffentlichen Arbeitsmarktpolitik sowie eine unzureichende politische und ökonomische Stabilität hemmen die Supply Chain-Leistung von Südafrika. Das Land muss seine relative Supply Chain-Leistung und Relevanz im südlichen Afrika halten und stärken, um wettbewerbsfähig zu bleiben.

Während Angola vor allem aber nicht ausschließlich durch Defizite in Basisfaktoren in der Supply Chain-Leistung gehemmt wird, werden Namibia und insbesondere Südafrika durch Defizite in Fortschrittsfaktoren in der Supply Chain-Leistung beschränkt. Angola steht vor Aufgaben, die Namibia und Südafrika längst bewältigt haben. Es wurde berichtet, dass Angola in einer Reihe von Faktoren auf Namibia und Südafrika schauen sollte, um Supply Chain-Leistung zu verbessern. Obwohl Namibia aufgrund der geringeren Volumina gegenüber Südafrika, in der Übernahme von neuen Technologien, hinter Südafrika bleiben wird, wurde berichtet, dass Namibia von einigen guten Beispielen in Südafrika 
lernen kann. Für Südafrika wurde berichtet, dass es zwar einige gute Beispiele auf dem afrikanischen Kontinent gibt, von denen Südafrika lernen könnte, die meisten guten Beispiele für Südafrika sind jedoch außerhalb des Kontinents zu finden.

\section{Relevanz der Ergebnisse für andere Länder und Regionen}

Die Fallstudienforschung zeigte eine Reihe von Erkenntnissen, die zwar in erster Linie für die drei ausgewählten Länder und das südliche Afrika von Relevanz sind, aber auch für andere Länder und Regionen von Wert sein können.

Erstens, wie die Fälle von Angola, Namibia und Südafrika zeigen, haben bisherige Entwicklungspfade das heutige Design von Supply Chain-Systemen im südlichen Afrika geprägt. Trotz Veränderungen im Zeitverlauf erklären historische Ausrichtungen von Supply Chain-Systemen ihre heutige Gestaltung. Auf der einen Seite können vergangene Gestaltungen förderlich für Supply Chain-Leistung sein; auf der anderen Seite erklären vergangene Gestaltungen noch heutige Schwachstellen und sogar wesentliche Engpässe. Aufgrund der Tatsache, dass Supply Chain-Systeme in vielen Ländern auf dem afrikanischen Kontinent gestaltet wurden, um ihre Kolonialmächte mit Rohstoffen zu versorgen und schließlich eine Entwicklung hin zu einer stärker unabhängigen Volkswirtschaft verhinderte, könnte dies auch auf andere Länder zutreffen.

Zweitens, wie der Fall von Angola zeigt, haben und halten nach wie vor Konflikte und Kriege eine Reihe von Ländern von einem Fortschreiten oder einer Aufrechterhaltung ihrer Stufe der Supply Chain-Leistung und ökonomischen Entwicklung $a b$ - während andere Länder die Chance haben, sich vorwärts zu bewegen. Diese Länder litten nicht nur unter verheerenden Kriegen, sondern auch unter einer Vernachlässigung der Entwicklung einer sich selbsttragenden Wirtschaftsstruktur. Aufgrund des Fehlens von einer Vielzahl von Faktoren müssen diese Länder zunächst Basisfaktoren von Supply Chain-Systemen bereitstellen, bevor fortgeschrittene Ziele erreicht werden können. Wie der Fall von Angola zeigt, sind Defizite in der physischen Infrastruktur offensichtlich; Defizite bestehen jedoch gleichermaßen in nicht-physischer Infrastruktur, wie formelle und informelle Institutionen. Obwohl der Krieg vor mehr als 16 Jahren endete, informelle Vorgehensweisen, die während des Krieges entstanden und sich etablierten, existieren heute nach wie vor. Ausbildung und Training ist ein weiterer Bereich, der häufig nicht ausreichend Aufmerksamkeit erhalten hat.

Drittens, wie der Fall von Angola, Namibia und Südafrika zeigte, wenngleich auf unterschiedlichen Niveaus, ist die Schienentransportleistung in allen drei Ländern unzureichend. Entweder sind Schienentransportdienstleistungen nicht verfügbar, die Leistungsniveaus und/oder Kosten sind nicht wettbewerbsfähig oder aber die Leistungsniveaus und/oder Kosten erfüllen nicht die minimal erforderlichen
Leistungsniveaus oder Kosten, um überhaupt einen Schienentransport zu ermöglichen. Dies verhindert zudem eine regionale Integration von Schienentransportsystemen im südlichen Afrika. Hinzu kommen Unterschiede in der Infrastruktur und im Equipment zwischen den Ländern. Wenngleich sich die Anforderungen an die Transporteigenschaften über die letzten Dekaden verändert haben und Schienentransport für viele Geschäftsvorfälle nicht mehr die bevorzugte modale Option darstellt, hat der Schienentransport in heutigen Supply Chain-Systemen nach wie vor eine wichtige Rolle zu spielen, insbesondere im multimodalen Langdistanz-Hinterland- und grenzüberschreitenden Transport. Unzureichender Schienentransport scheint nicht nur in den drei ausgewählten Ländern der Fall zu sein und deren inländische und grenzüberschreitende Transport- sowie Supply Chain-Leistung zu beschränken, sondern in vielen weiteren Ländern auf dem afrikanischen Kontinent ebenso. Wichtig hervorzuheben ist an dieser Stelle, dass eine Wiederbelebung von Schienentransportsystemen nicht lediglich die Rekonstruktion und Entwicklung von Infrastruktur umfasst, sondern die Wiederherstellung und Entwicklung von angemessen funktionierenden Betriebsabläufen gleichermaBen.

Viertens, eine illegale Umgehung von Straßentransportregulationen ist ein Thema in allen drei Ländern. Der starke Wettbewerb in der Straßentransportbranche zusammen mit einer unzureichenden Anwendung des geltenden Rechts, oder unzureichenden Gesetzgebung, schaffen Anreize für eine Umgehung der öffentlichen Regulation. Illegale Umgehung von Transportregulationen erschwert eine nachhaltige Entwicklung des Transportsektors und, demzufolge, eine nachhaltige Entwicklung von Supply Chain-Leistung. Es gibt Anzeichen, dass eine illegale Umgehung von Straßentransport-Regulation ebenso ein Thema in anderen Ländern auf dem afrikanischen Kontinent ist.

Fünftens, wie die Fälle von Angola, Namibia und Südafrika zeigen, werden wesentliche Teile von Supply Chains von politischen Entscheidungsträgern oft als ein Mittel zur Erreichung von nationalen ökonomischen Entwicklungszielen gesehen. Aufgrund des hohen Beteiligungsgrades des öffentlichen Sektors in Supply Chains in Namibia und Südafrika, wie in Häfen und Schienentransportsystemen, und allgemeiner Infrastruktur, wie Elektrizitäts- und Wasserversorgung, wird die Verantwortung oft bei dem öffentlichen Sektor gesehen. Darüber hinaus besteht in diesen beiden Ländern ein hoher Grad des Eingriffs in den privaten Sektor. In beiden Ländern fühlt sich der öffentliche Sektor verantwortlich, Unterschiede aus der Vergangenheit abzumildern beziehungsweise zu beseitigen - einerseits durch die aktive Rolle als Supply Chain-Akteur und andererseits durch öffentliche Regulation. Dennoch gibt es Kontroversen über die angemessene Rolle und den Grad der Beteiligung und Intervention des öffentlichen Sektors in Supply Chains. In Angola ist die Rolle des öffentlichen Sektors als direkter Akteur im Betrieb von Supply Chains geringer als in Namibia und Südafrika. Den- 
noch besteht auf der einen Seite ein erheblicher Grad der öffentlichen Intervention und auf der anderen Seite gibt es Defizite in der notwendigen öffentlichen Regulation des privaten Sektors. Wenngleich unter anderen Umständen, infolge von Defiziten in der ökonomischen Entwicklung, könnte dieses Dilemma ebenso auch in anderen Ländern vorzufinden sein.

Sechstens, wie die Fälle von Namibia und Südafrika und ebenso, seit der Rezession, von Angola zeigen, bringt der Mangel an finanziellen Ressourcen eine Rivalität zwischen auf der einen Seite sozio-ökonomischen Faktoren, wie einer allgemeinen Infrastruktur, und auf der anderen Seite Supply Chain-Faktoren. Aufgrund von Defiziten in der ökonomischen Entwicklung müssen diese Regierungen einen Mittelweg finden zwischen Ausgaben und Entwicklung von sozialen und ökonomischen Bereichen. Dies ist wichtig zu betonen, nicht nur aufgrund der Tatsache, dass ökonomische Entwicklung als ein primäres Ziel von Ländern angesehen werden sollte, sondern auch aufgrund der Tatsache, dass das Niveau der ökonomischen Entwicklung einen Einfluss auf die Supply Chain-Leistung eines Landes hat. Dies könnte ebenso auf Länder auf einer niedrigen ökonomischen Entwicklungsebene mit erheblichen Herausforderungen in der Supply Chain-Leistung zutreffen.

Siebtens, wie die zentralen und angrenzenden Fälle dieser Fallstudienarbeit zeigen, ist die ökonomische Integration deutlich unter ihrem Potenzial und alle drei Länder könnten von einem höheren Grad der ökonomischen Integration einen Nutzen ziehen. Jedoch hemmt eine Reihe von Faktoren eine höhere Integration und damit weitere Entwicklung des regionalen Supply Chain-Systems im südlichen Afrika. So stellen Umsätze vom Handel nach wie vor eine wichtige Quelle von öffentlichen Einnahmen in einigen Ländern dar. Eine Freihandelszone gibt es nach wie vor nicht im südlichen Afrika. Als Folge daraus müssen Transporte an Grenzübergängen für Grenzkontrollen stoppen. Wenngleich eine Abschaffung von Grenzkontrollen, zum jetzigen Zeitpunkt, nicht angemessen wäre, besteht ein erhebliches Verbesserungspotenzial hinsichtlich der Infrastruktur, der Prozesse, der Gesetzgebung und Einhaltung von Gesetzen an Grenzübergängen. Darüber hinaus erfordern Unterschiede in der Gesetzgebung und Standardisierung über Länder zusätzliche Vorgehensweisen zur Einhaltung der Gesetze. Eine Harmonisierung und Standardisierung ist noch am Anfang im südlichen Afrika. So sollten zum Beispiel Zolldokumente, Bruttogewichtsbeschränkungen und Anforderungen an die technische Qualität von Fahrzeugen über die Länder harmonisiert werden. Unterschiede in den Spurweiten und Antriebsarten von Schienentransport erfordern ein Wechsel von Fahrzeugen und Besatzung und erschweren damit einen grenzüberschreitenden Schienentransport. Schließlich berichteten Interviewpartner, dass es Gespräche über die Einführung einer gemeinsamen Währung in der SADC gäbe. Jedoch muss dabei berücksichtigt werden, dass diese Länder noch unterschiedlicher sind als die Länder der
Euro-Zone. Aus diesem Grund scheint eine einzige, gemeinsame Währung in der SADC wenig Aussicht auf Erfolg zu versprechen.

Schließlich, wie die Fälle von Namibia und Südafrika offenlegen, im grenzüberschreitenden Transport treffen eine große Zahl von Akteuren, das heißt Menschen und Organisationen aus unterschiedlichen Ländern, mit unterschiedlichem kulturellem Hintergrund sowie unterschiedlichem Ausbildungshintergrund zusammen. Dies führt dazu, dass die Menschen sich unterschiedlich verhalten. Aufgrund der Notwendigkeit für gemeinsame Standards in Supply Chains kann dies jedoch zu einem Aufeinandertreffen von unterschiedlichen Denk-, Vorgehens- und Verhaltensweisen führen. Diese Probleme liegen zumindest kurzfristig außerhalb der Kontrolle einzelner Akteure, Organisationen und Ländern und kann die Supply Chain-Leistung beschränken. Wie der Fall von Angola offenlegt, wurden diese Verhaltensfaktoren sukzessiv über einen langen Zeitraum entwickelt und können nicht über Nacht verändert werden. Entschlossene und langfristige Maßnahmen scheinen notwendig, um notwendige Verbesserungen hervorzurufen. Das heißt, dass Veränderungen in der Supply Chain-Leistung in einem Land nicht ausschließlich auf Maßnahmen innerhalb eines Landes beruhen, sondern ebenso Maßnahmen außerhalb des Landes erfordern. Verbesserungen in der Supply Chain-Leistung in einem Land erfordern ebenso Verbesserungen der Leistung in anderen Ländern.

\section{Beitrag des Projektes und Anknüpfungspunkte}

Es war die Erwartung, mit dieser Arbeit einen Beitrag zur laufenden Forschung von Engpässen von Supply Chain-Leistung in den drei ausgewählten Ländern aber darüber hinaus auch mit Blick auf andere Länder und Regionen zu leisten. Diese Arbeit hat wesentliche Grundlagen gelegt, erfordert aber weitere Forschung insbesondere zu den folgenden drei Aspekten:

Erstens, diese Arbeit hat Engpässe von Supply Chain-Leistung identifiziert und empfohlene Maßnahmen zur Verbesserung, mögliche Hindernisse sowie Verantwortlichkeiten ermittelt. Dennoch, Engpässe, Maßnahmen, Verantwortlichkeiten und Hindernisse können von Region zu Region in einem Land variieren. Um eine detailliertere Ebene zu erreichen, ist mehr lokales und spezialisiertes Wissen und Input notwendig.

Zweitens, die Datengrundlage sowie die Schlussfolgerungen beziehen sich auf den Zeitpunkt der Datenerhebung und -analyse. Faktoren, die Engpässe in der Vergangenheit darstellten, könnten keine Engpässe in der Zukunft mehr sein. Ebenso könnte es sein, dass Maßnahmen zur Verbesserung nicht mehr geeignet sind, um Engpässe in der Zukunft abzumildern oder zu beseitigen. Das bedeutet, dass die Ergebnisse dieser Arbeit nicht mehr und nicht weniger als eine Momentaufnahme darstellen und Folgeaktivitäten erfordern. 
Drittens, während die Methode eine Verbesserung gegenüber methodischen Ansätzen darstellt, die sich ausschließlich auf Leistungswerte beziehen, bringt die Messung von Wichtigkeitswerten und die Kombination von Wichtigkeitsund Leistungswerten ebenso Herausforderungen mit sich. So stellte die Messung der jeweiligen Wichtigkeits- und Leistungswerte hohe Anforderungen an die Anzahl der Umfrageantworten. Basierend auf der Beschreibung des methodischen Vorgehens, der Darstellung der Ergebnisse dieser Arbeit sowie den Einschränkungen könnte weitere Forschung der Verbesserung und Anwendung des methodischen Instrumentes gewidmet werden. Wichtig zu erwähnen ist, dass die Interviewpartner die persönlichen Interviews als einen wertvollen aber bisher zu gering eingesetzten Ansatz in der Forschung zu Logistik und Supply Chains im südlichen Afrika halten.

Die Dissertation steht unter der folgenden URL zum Download bereit: https://www.zhb-flensburg.de/dissert/ hofmann/

\section{Literatur}

Arvis, Jean-François; Saslavsky, Daniel; Ojala, Lauri; Shepherd, Ben; Busch, Christina; Raj, Anasuya; Naula, Tapio (2016): Connecting to Compete 2016. Trade Logistics in the Global Economy. The World Bank. Washington, DC.

Feige, Irene (2007): Transport, Trade and Economic Growth - Coupled or Decoupled? An Inquiry into Relationships between Transport, Trade and Economic Growth and into User Preferences Concerning Growth-oriented Transport Policy. Berlin, Heidelberg: Springer.

Hall, Peter; McCalla, Robert J.; Comtois, Claude; Slack, Brian (2011): Introduction. In Peter Hall, Robert J. McCalla,
Claude Comtois, Brian Slack (Eds.): Integrating Seaports and Trade Corridors. Farnham, Surrey; Burlington, VT: Ashgate, pp. 1-10.

Hausmann, Ricardo; Hidalgo, César A.; Bustos, Sebastián; Coscia, Michele; Simoes, Alexander; Yildirim, Muhammed A. (2013): The Atlas of Economic Complexity. Mapping paths to prosperity. Cambridge, MA: The MIT Press.

Hausmann, Ricardo; Klinger, Bailey; Wagner, Rodrigo (2008): Doing Growth Diagnostics in Practice: A ,Mindbook'. CID Working Paper No. 177. Center for International Development at Harvard University.

Hausmann, Ricardo; Rodrik, Dani; Velasco, Andrés (2008): Growth Diagnostics. In Narcís Serra, Joseph E. Stiglitz (Eds.): The Washington Consensus Reconsidered. Towards a New Global Governance. Oxford: Oxford University Press, pp.324-355.

Saslavsky, Daniel; Shepherd, Ben (2012): Facilitating International Production Networks. The Role of Trade Logistics. Policy Research Working Paper No. 6224. The World Bank.

UNDP (United Nations Development Programme) (2016): Human Development Report 2016. New York.

UNIDO (United Nations Industrial Development Organization); UNCTAD (United Nations Conference on Trade and Development): Economic Development in Africa Report 2011 (2011). Fostering Industrial Development in Africa in the New Global Environment. The United Nations. New York and Geneva.

WEF (World Economic Forum) (2017): The Global Competitiveness Report 2017-2018. Geneva. 